\title{
InAs photodiode for low temperature sensing
}

\author{
X. Zhou*, J. S. Ng and C. H. Tan** \\ Department of Electronic and Electrical Engineering, University of Sheffield, Mappin Street, \\ S1 3JD, U.K.
}

\begin{abstract}
We report on the evaluation of InAs photodiodes and their potential for low temperature sensing. InAs n-i-p photodiodes were grown and analyzed in this work. Radiation thermometry measurements were performed at reference blackbody temperatures of 37 to $80{ }^{\circ} \mathrm{C}$ to determine photocurrent and temperature error. The uncooled InAs photodiodes, with a cutoff wavelength of $3.55 \mu \mathrm{m}$, detect a target temperature above $37^{\circ} \mathrm{C}$ with a temperature error of less than $0.46^{\circ} \mathrm{C}$. When the photodiode was cooled to $200 \mathrm{~K}$, the temperature error at $37^{\circ} \mathrm{C}$ improves by 10 times from 0.46 to $0.048^{\circ} \mathrm{C}$, suggesting the potential of using InAs for human temperature sensing.
\end{abstract}

Keywords: Radiation thermometry, temperature measurement, InAs photodiodes

\section{INTRODUCTION}

Radiation thermometry is used to monitor the temperature of an object without physical contact, by measuring the emitted energy from the object over a specific wavelength range, to determine its blackbody temperature. They have been widely used in steel [1], glass [2] and plastics industry [3]. Traditionally thermal detectors, such as thermopiles and pyroelectrics, or photon detectors, have been used. They respond to radiation over a broad spectrum by employing a wide band absorbent materials, thus can detect to low temperature range [4]. However, they suffer from low detectivity and have long response time. Hence, they are not suitable for high speed measurements. Instead of heating up a material in response to incident radiation, photon detectors experience an electronic state change when the incident wave is absorbed. Photon detectors present much faster response speed and higher detectivity than thermal detectors. Unlike the thermal detectors, the photon detectors have a limited wavelength response range determined by the band gap energy of the materials. Therefore semiconductors with different band gaps are normally employed to cover different temperature ranges.

In practical measurements, a major challenge is the uncertainty of target emissivity which depends on the material physical property and its surface conditions. The emissivity factor is the ratio of the spectral radiance from an object to that of a blackbody at the same temperature. A small uncertainty in the emissivity will lead to significant error in radiation thermometry, particularly at longer infrared wavelengths. Fortunately at shorter wavelengths the rate of change in the spectral radiance as a function of temperature increases more rapidly, leading to a smaller temperature error [5]. In addition to the smaller error, from semiconductor physics it is well known that the short wavelength detectors have lower leakage current and hence lower shot noise. Si and InGaAs photodetectors have been widely used as high temperature sensors in radiation thermometry with a working wavelength of $0.9 \mu \mathrm{m}$ and $1.6 \mu \mathrm{m}$, respectively. Due to the non-linear spectral power of Planck's law, they are limited to lower temperature limits of $400{ }^{\circ} \mathrm{C}$ for $\mathrm{Si}[5]$ and $150{ }^{\circ} \mathrm{C}$ for standard $\mathrm{InGaAs}$ [6]. As the target temperature decreases, the peak in spectral radiance shifts to longer wavelengths. Long wavelength operation needs to be selected for lower temperature measurements or to account for material optical transmission properties. By changing the alloy composition during the growth and optimization in fabrication process, InGaAs can be used to detect IR radiation up to $2.6 \mu \mathrm{m}$. Yoon et al. [7] demonstrated that an extended InGaAs with a $2.5 \mu \mathrm{m}$ cut-off wavelength and cooled to $-85^{\circ} \mathrm{C}$ can be used to sense a $50{ }^{\circ} \mathrm{C}$ target with a noise-equivalent temperature difference (NETD) $<3 \mathrm{mK}$. Lead salt alloys PbSe photoconductors with cut-off wavelength $5.0 \mu \mathrm{m}$ have been widely used for sensing object above $30^{\circ} \mathrm{C}$ [8]. However, it suffers from high $1 / \mathrm{f}$ noise and growth issues for availability of large scale arrays. Narrow band gap materials such as InSb are also used in a radiation thermometer for medium and low temperature range $[9,10]$. Unfortunately, InSb detectors suffer from high dark current and needs to be cooled to liquid-nitrogen temperature.

InAs photodiodes have recently been demonstrated as a promising material for infrared sensing [11]. With a band gap of $0.36 \mathrm{eV}$, InAs photodiodes exhibit a cut-off wavelength of $3.55 \mu \mathrm{m}$ at room temperature, providing a high detectivity around $3.39 \mu \mathrm{m}$. Therefore, InAs photodiodes is a potential candidate for low temperature thermometer or thermal imager without cooling or thermoelectric cooler. Recently Hobbs et al. [12] demonstrated that an avalanche photodiode can

*x.zhou@ sheffield.ac.uk; phone +44 (0) 114222 5410; fax +44 (0) 1142225143

**c.h.tan@sheffield.ac.uk; phone +44 (0) 114222 5144; fax +44 (0) 1142225143

Sensors, Systems, and Next-Generation Satellites XIX, edited by Roland Meynart, Steven P. Neeck, Haruhisa Shimoda Proc. of SPIE Vol. 9639, 96390V · C 2015 SPIE · CCC code: 0277-786X/15/\$18 · doi: 10.1117/12.2197343 
provide significant signal to noise ratio improvement in radiation thermometry. InAs has also been demonstrated as an excellent avalanche photodiode that operates with single carrier multiplication to produce negligible excess noise, presenting a huge potential further improvement for infrared imaging [13, 14]. Moreover, Sandall et al. have reported linear arrays of $1 \times 128$ InAs avalanche photodiodes [15]. Therefore future IR radiation thermometers could potentially be enhanced using discrete and arrays of InAs photodiodes or avalanche photodiodes. In this work, we performed a detailed characterization of InAs photodiode and evaluated its potential for low temperature sensing.

\section{DEVICE STRUCTURE AND CHARACTERISATION}

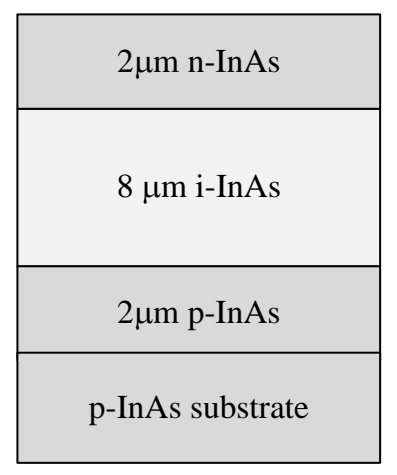

(a)

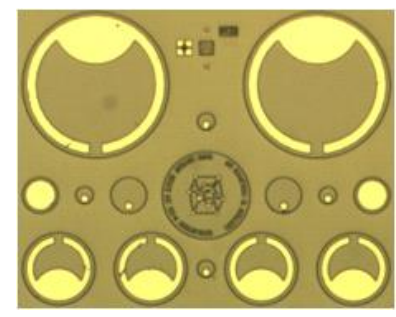

(b)

Figure 1. (a)The schematic structure and (b) top view image of fabricated device of various sizes

The InAs wafer studied in this work was grown by Molecular Organic Chemical Vapour Deposition (MOCVD). The structure was grown on 2" p-type InAs substrates. The structure comprised of a $2 \mu \mathrm{m} p+$ layer $\left(1 \times 10^{18} \mathrm{~cm}^{-3}\right)$ followed by an intrinsic region thicknesses of $8 \mu \mathrm{m}$ and then a $2 \mu \mathrm{m} \mathrm{n}+$ layer $\left(1 \times 10^{18} \mathrm{~cm}^{-3}\right)$, as shown in Figure 1(a). Ti/Au metal with thickness of 20/200 nm was deposited to form top and bottom ohmic contacts. The sample was fabricated using wet chemical etchants [16] of 1:1:1 (phosphoric acid: hydrogen peroxide: deionized water) etch, followed by a finishing etch of 1:8:80 (sulphuric acid: hydrogen peroxide: de-ionized water), to define the mesa diodes with diameters of 420, 220, 120 and $70 \mu \mathrm{m}$ in Figure 1(b).

Current-voltage (I-V) measurement was performed using a Keithley 236 source measure unit. The dark current densities from on-wafer diodes with different diameters are in good agreement (not shown here), indicating that bulk dark current dominates at room temperature. Figure 2(a) shows the temperature dependence of dark current of InAs photodiodes with a diameter of $420 \mu \mathrm{m}$. The reverse dark current reduces $\sim 3$ orders of magnitude from 295 to $200 \mathrm{~K}$ which is similar to the trend in the work by Ker et al. [17]. The capacitance-voltage (C-V) measurements were also performed on these devices to study the material background doping. To perform accurate measurement, the detector was cooled to $77 \mathrm{~K}$ to reduce errors induced by the high dark current. Figure 2(b) shows the capacitances and depletion width of InAs with different sizes as a function of reverse bias. C-V results suggest this wafer is not fully depleted at zero bias and has an unintentional doping level of $6 \times 10^{14} \mathrm{~cm}^{-3}$ in the i-InAs layer. 

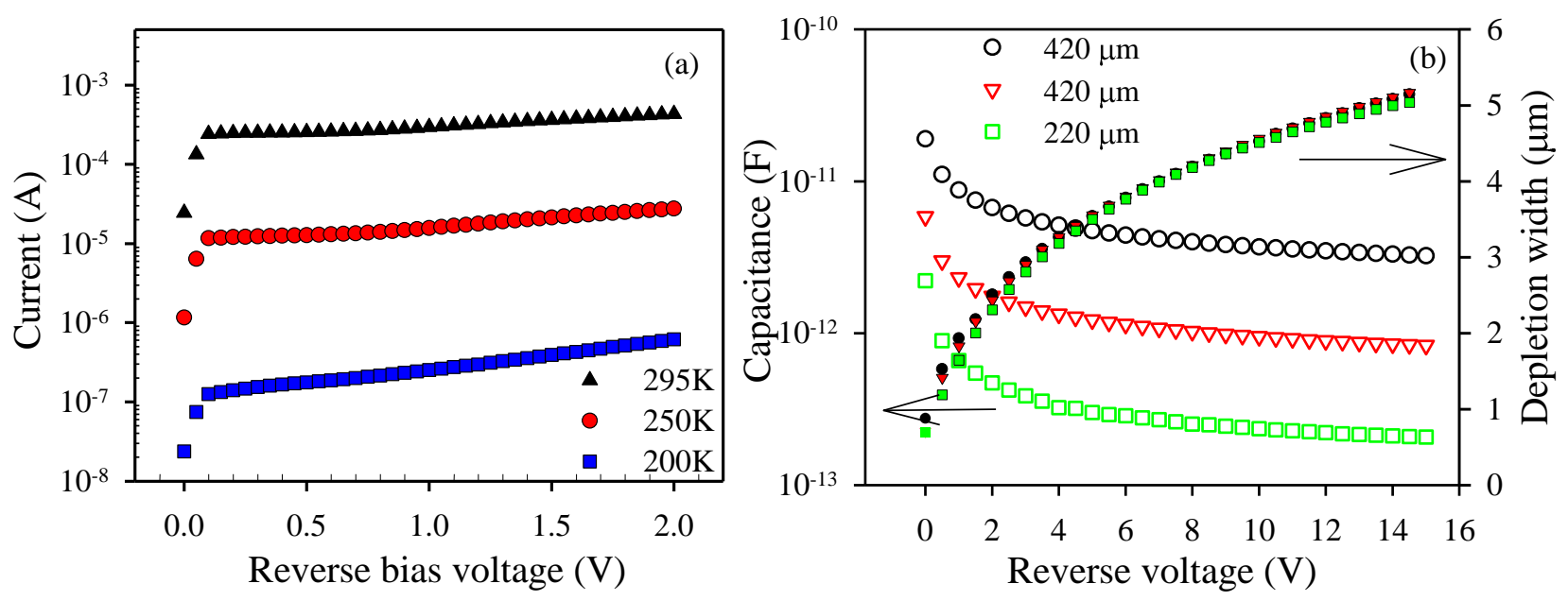

Figure 2. (a) Temperature dependence of dark current of the InAs photodiodes with diameter of $420 \mu \mathrm{m}$ and (b) Capacitance and depletion width of InAs photodiodes with different diameters at $77 \mathrm{~K}$

The spectral response on InAs was also performed in this work. Figure 3(a) shows the room temperature responsivity comparison of our InAs photodiodes and commercial InAs detectors. The responsivity was deduced using normalized spectral response obtained from the FTIR and the peak responsivity measured using a $T_{B B}=800{ }^{\circ} \mathrm{C}$. Responsivity values at wavelengths of 633, 1520 and $2004 \mathrm{~nm}$ were cross-checked using $\mathrm{CW}$ lasers at the respective wavelengths. At $0 \mathrm{~V}$, the InAs diode shows a peak responsivity of $1.28 \mathrm{~A} / \mathrm{W}$ at $3.35 \mu \mathrm{m}$ and a cut-off wavelength (at $50 \%$ of peak response) of 3.55 $\mu \mathrm{m}$, giving $\sim 48 \%$ external quantum efficiency (EQE) across the whole spectrum. The responsivity from our InAs diode is higher than that from Hamamatsu InAs diode [18] and lower than Judson diode [19] at longer wavelengths. However, this external quantum efficiency can be improved by increasing the depletion width. The depletion width at $0 \mathrm{~V}$ is only $0.95 \mu \mathrm{m}$ and increases with reverse bias, as shown in Figure 2(b). Based on the measured dark current and responsivity at $0 \mathrm{~V}$, our InAs detectivity values were calculated and compared to those from Hamamatsu and Judson InAs, as shown in Figure 3(b). Despite lower responsivity and shorter cut-off wavelength than Judson InAs, our diode shows higher detectivity than commercial InAs detectors. This is due to lower detector dark current than that of Judson InAs and higher responsivity than that from Hamamatsu InAs.
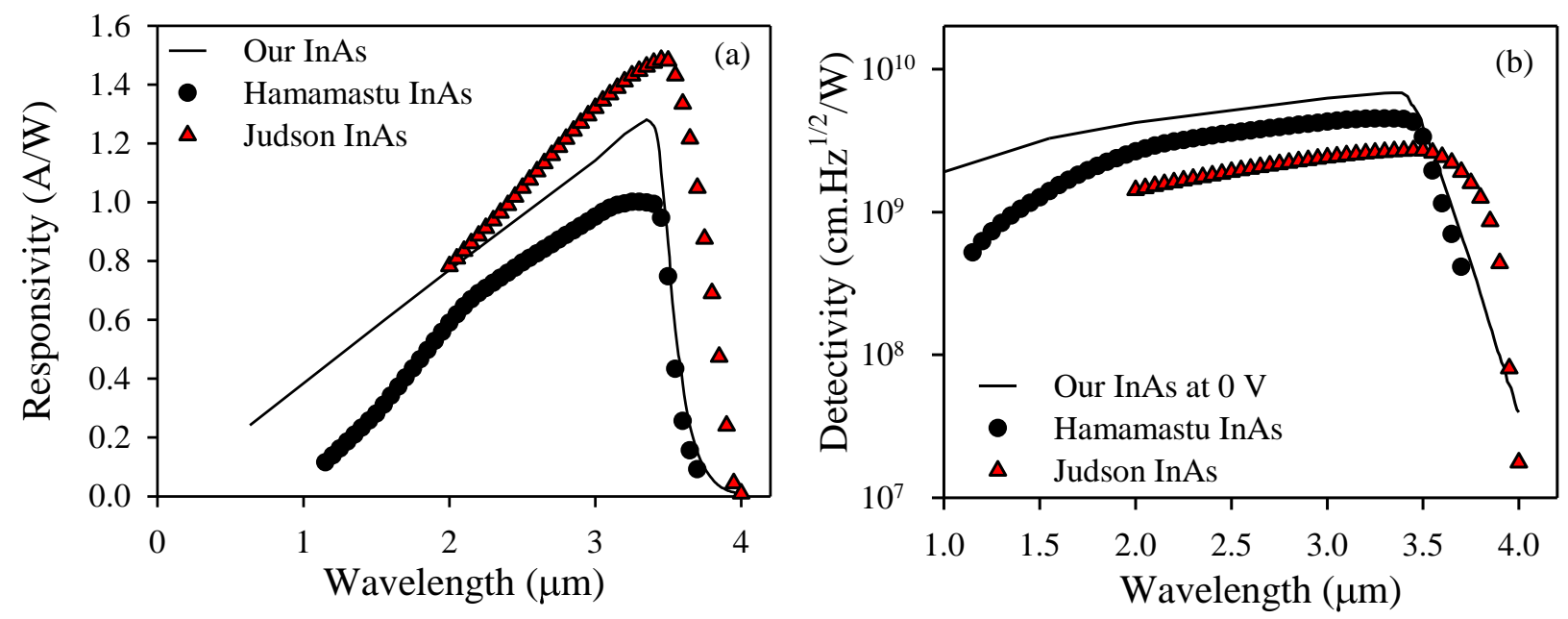

Figure 3. (a) Responsivity and (b) detectectivity of our InAs photodiode and commercial InAs detectors at room temperature 


\section{RADIATION THERMOMETRY}

The temperature dependence of thermometry measurement was performed using the cooling system illustrated in Figure 4. A LANDCAL P80P blackbody source, with an aperture diameter of $7.5 \mathrm{~mm}$, was set at temperatures between 37 and $80{ }^{\circ} \mathrm{C}$ with the detector temperature set to 295 and $200 \mathrm{~K}$ in these measurements. The photocurrent was measured using a phase sensitive detection (PSD) method, where the light signal from a blackbody source is modulated at a frequency of $420 \mathrm{~Hz}$. The device under test (DUT) was placed at a distance of $300 \mathrm{~mm}$ from the blackbody source and a ZnSe lens with $50 \mathrm{~mm}$ focal length was used to focus light onto the detector. We noted that the optical spot size is not fully optimized as it is larger than the device area. The output photocurrent was amplified by a transimpedance amplifier (TIA) with an overall gain of $10^{6}$ and then measured using a SR830 lock-in amplifier. In this work, the device with $420 \mu \mathrm{m}$ diameter was used for thermometry measurement.

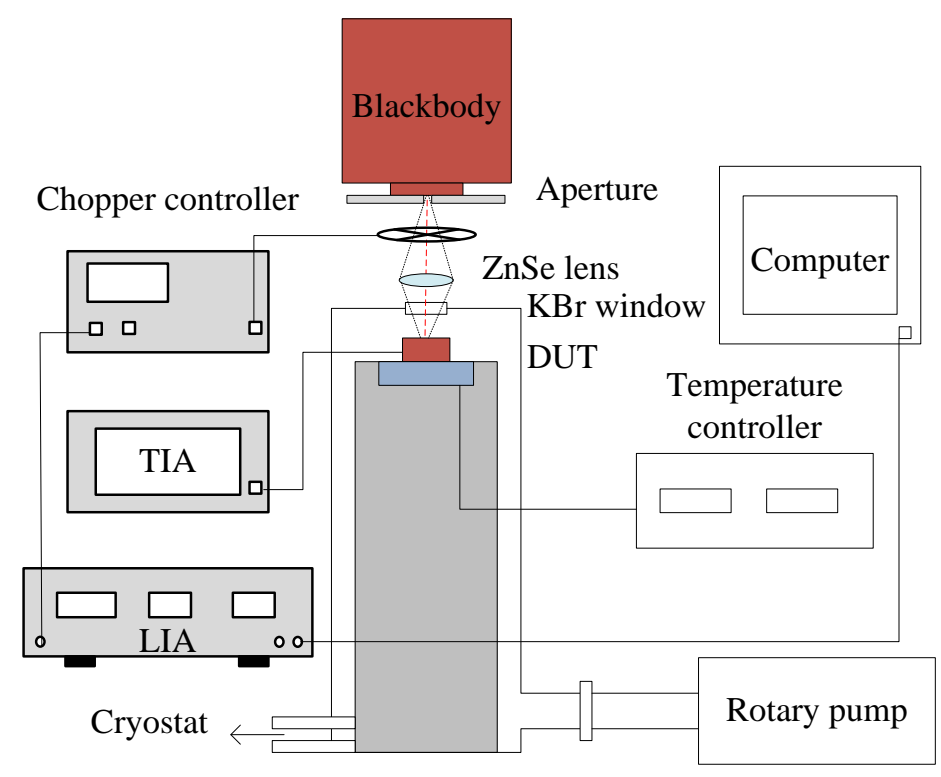

Figure 4. Radiation thermometry setup

As device dark current increases much faster than the responsivity with increased reverse bias, the radiation thermometry measurements were performed at $0 \mathrm{~V}$ to minimize the influence of dark current. Figure 5 shows the mean photocurrent of the InAs photodiode. As $\mathrm{T}_{\mathrm{BB}}$ increases, the photocurrent increases due to higher incident photon flux. As the detector temperature reduces from 295 to $200 \mathrm{~K}$, the output photocurrent is reduced by around $50 \%$ at all $\mathrm{T}_{\mathrm{BB}}$ range. This is due to the cut-off wavelength shifting to lower wavelengths when the detector was cooled to lower temperature. The normalized InAs spectrum at detector temperatures of 295 to $200 \mathrm{~K}$ is shown in Figure 6. From Planck's curve of radiated energy from blackbody, the majority of radiated photons from the blackbody at $37-80^{\circ} \mathrm{C}$ are at longer wavelengths. The smaller overlap between the spectral response at lower detector temperature and blackbody radiated spectrum results in reduced photocurrent. 


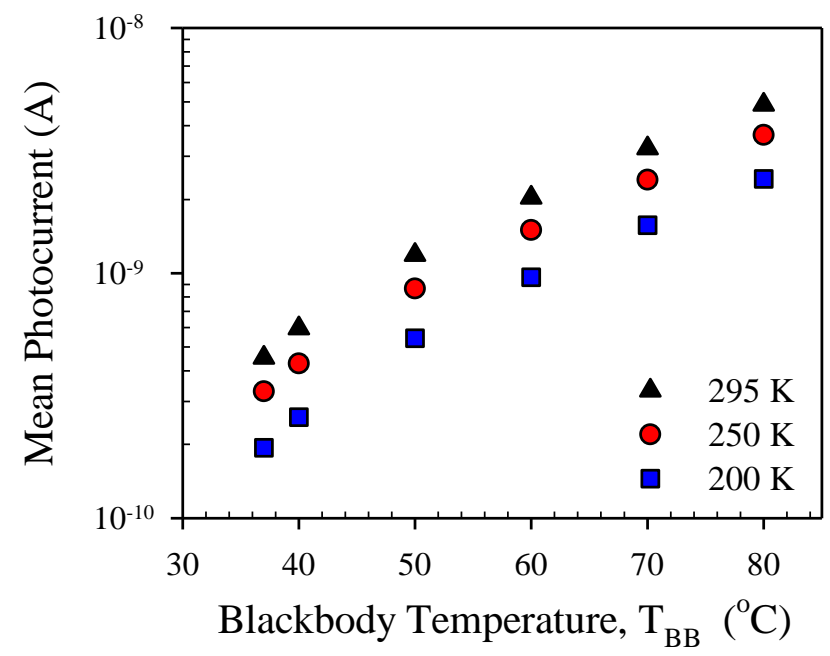

Figure 5. Mean Photocurrent of the InAs photodiodes at different detector temperatures at $0 \mathrm{~V}$

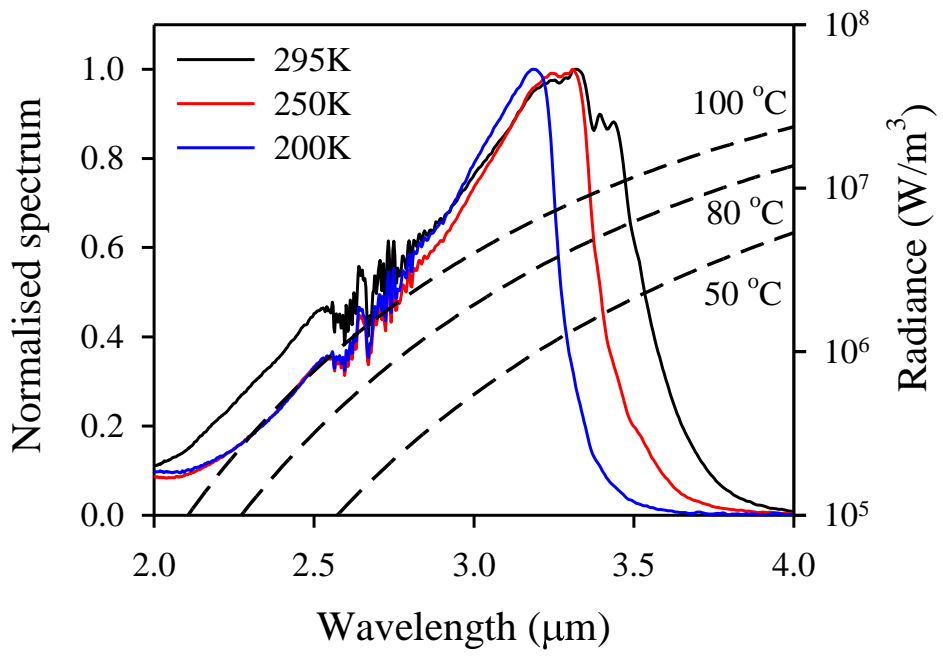

Figure 6. Normalized spectrums of the InAs photodiodes at different detector temperatures of 295 to $200 \mathrm{~K}$ and blackbody radiance at $\mathrm{T}_{\mathrm{BB}}=50,80$ and $100{ }^{\circ} \mathrm{C}$

\section{TEMPERATURE ERROR ANALYSIS}

The output signal-to-noise ratio ( $S N R$ ) was taken the ratio of the mean output photocurrent, $\left\langle I_{p h}\right\rangle$ to the standard deviation of output photocurrent, $\sigma\left(I_{p h}\right)$. In order to evaluate the detector performance as a radiation thermometer, the temperature error can be calculated. This value was calculated from the ratio of percentage error of output photocurrent to the percentage in output for a $1{ }^{\circ} \mathrm{C}$ rise in the target temperature, known as percent-per-degree. The percentage error of the output, \%output error , is related to $S N R$ and is expressed as

$$
\%_{\text {output error }}=100 \times \frac{\sigma\left(I_{p h}\right)}{\left.<I_{p h}\right\rangle} .
$$

The Percent-per-Degree, which is given by [20]

$$
\% /{ }^{\circ} \mathrm{C}=100 \times \frac{c_{2}}{\lambda T^{2}} .
$$


where $c_{2}$ is Planck's second constant $(1.4388 \mathrm{~cm} \cdot \mathrm{K}), \lambda$ is the effective operational wavelength of the thermometer, and $T$ is the object temperature in Kelvin. The effective wavelength is derived from the gradient of the natural logarithm of output photocurrent plotted as a function of 1/T [21]. This method is based on the Wien's law approximation to Planck's Law and is useful to model broad band radiations as a single monochromatic wavelength [22].

The mean $S N R$ and temperature errors at different detector temperatures are shown in Figure 7. Following the trend of photocurrent, the $S N R$ increases with blackbody temperatures. Despite the drop in photocurrent, the $S N R$ increases with decreasing detector temperature. This is because as the temperature is reduced the dark current drops much more rapidly than the photocurrent as illustrated in Figure 2 and Figure 5. For instance, the photocurrent drops only 1/3 while the dark current drops by more than 20 times at $250 \mathrm{~K}$ relative to the values at room temperature. The temperature error of InAs photodiodes in Figure 7 reduces slowly from 250 to $200 \mathrm{~K}$. It is of particular interest to note that the device dark current reduces by 1000 times when the diode is cooled from room temperature to $200 \mathrm{~K}$. The temperature error improves by 10 times from 0.46 to $0.048^{\circ} \mathrm{C}$ at a $\mathrm{T}_{\mathrm{BB}}=37^{\circ} \mathrm{C}$, confirming the potential of using InAs for low temperature sensing, including human temperature sensing. The amplifier noise is around $125 \mathrm{nV} / \mathrm{Hz}^{1 / 2}$ (obtained through separate measurements using an FFT spectrum analyzer) which gives an equivalent noise current level of $\sim 50 \mathrm{nA}$. As detector temperature reduces, the noise from dark current reduces such that the amplifier noise becomes dominant at $200 \mathrm{~K}$. Therefore, a lower noise amplifier will allow the InAs photodiodes to be used for performing more accurate at lower temperature. An alternative approach is to use a smaller device to reduce the detector dark current. For instance halving the detector diameter will reduce the dark current by 4 times so that the dark current reduces to $\sim 5 \mathrm{nA}$ at $200 \mathrm{~K}$. Provided that the optical coupling can be optimized, it should be possible to operate in the avalanche mode which will provided a higher SNR and lower temperature error in future work.

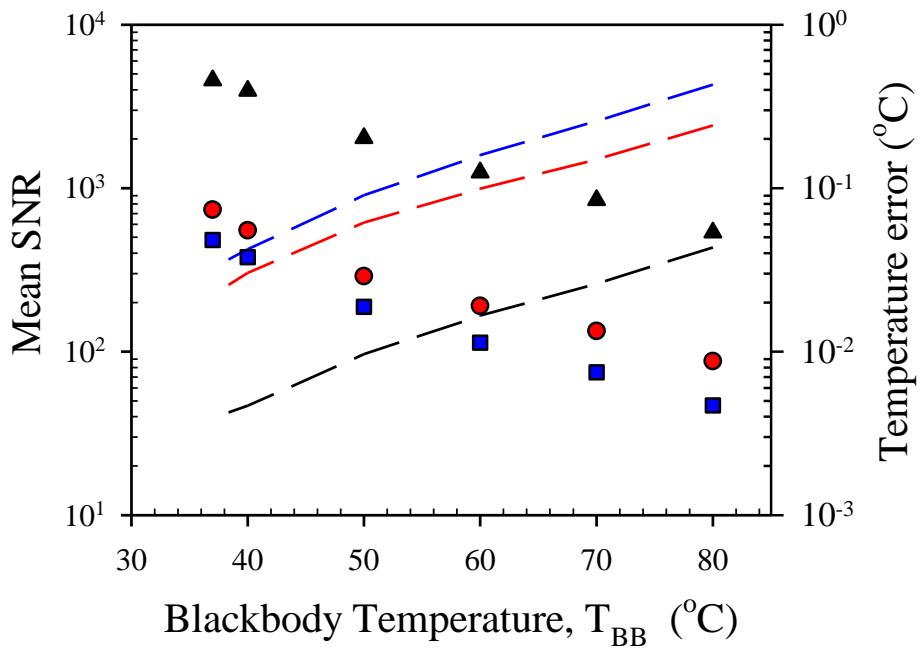

Figure 7. SNRs (lines) and temperature errors (symbols) and of the InAs photodiodes at detector temperatures of $295 \mathrm{~K}$ (Black), $250 \mathrm{~K}$ (Red) and $200 \mathrm{~K}$ (Blue)

\section{CONCLUSION}

In conclusion, the electrical and optical performance of InAs photodiodes have been characterized. They show bulk dark current mechanism and high quantum efficiency at room temperature. Compared with commercial InAs detectors, our InAs showed higher detectivity values within the spectral range. We have also studied the performance of InAs photodiodes for use in radiation thermometry. For uncooled operation, a target temperature of above $37{ }^{\circ} \mathrm{C}$ can be measured with a temperature difference of $\pm 0.46^{\circ} \mathrm{C}$. This temperature error can be reduced to $0.048{ }^{\circ} \mathrm{C}$ at a device temperature of $200 \mathrm{~K}$, demonstrating the huge potential for low temperature sensing. 


\section{ACKNOWLEDGMENT}

The authors would like to thank the EPSRC National Centre for III-V Technologies at The University of Sheffield for wafer growth and access to fabrication facilities. This work was supported by the UK Engineering and Physical Sciences Research Council (EPSRC) (grants EP/K001469/1, EP/H031464/1 and EP/I010920/1) and European Space Agency (Contract No.4000107110/12/NL/CBi).

\section{REFERENCES}

[1] Peacock, G. R., "Ratio radiation thermometry thermometers in hot rolling and galvannealing of steel strip," Proc. AIP 684, 789-794 (2003).

[2] Richmond, J. C. and Dewitt, D. P., [Applications of Radiation Thermometry], American Society for Testing and Materials, Philadelphia, 67-73 (1985).

[3] Esser, K., Haberstroh, E., Husgen, U. and Weinand, D., "Infrared radiation in the processing of plastics: Precise adjustment-the key to productivity," Advances in Polymer Technology 7(2), 89-128 (2003).

[4] Zhang, Z. M., Tsai, B. K. and Machin, G., [Radiometric temperature measurements. II. Applications], Elsevier Inc., USA, 2-54 (2010).

[5] Quinn, T. J., [ Temperature], Academic Press, London, UK, 332-431 (1990).

[6] Hill, K.D. and Woods, D. J., "Characterizing the NRC blackbody sources for radiation thermometry from $150{ }^{\circ} \mathrm{C}$ to $962{ }^{\circ} \mathrm{C}$, , Int. J. Thermophys. 30(1), 105-123 (2009).

[7] Yoon, H. W. and Eppeldauer, G. P., "Measurement of thermal radiation using regular glass optics and short-wave infrared detectors," Optical Express 16(2), 937-940 (2008).

[8] Meca, F. J. M., Quintas, M. M., Sánchez, F. J. R. and Sainz, P. R., "Infrared temperature measurement system using photoconductive PbSe sensors without radiation chopping," Sensors and Actuators A: Physical 100(2-3), 206-213 (2002).

[9] McEvoy, H. C., Lowe, D. H. and Owen, M., "The NPL InSb-based radiation thermometer for the medium temperature range $\left(<962{ }^{\circ} \mathrm{C}\right), "$ Int. J. Thermophys 28(6), 2067-2075 (2007).

[10] Ishii, J. and Ono, A., "Low-Temperature infrared radiation thermometry at NMIJ," Proc. AIP 684, 657-662 (2003).

[11] Ker, P. J., Marshall, A. R., David, J. P. R. and Tan, C. H., "Low noise high responsivity InAs electron avalanche photodiodes for infrared sensing," physica status solidi (c) 9(2), 310-313 (2012).

[12] Hobbs, M. J., Tan, C. H. and Willmott, J. R., "Evaluation of phase sensitive detection method and Si avalanche photodiode for radiation thermometry," JINST. 8(3), P03016 (2013).

[13] Marshall, A. R. J., Tan, C. H., Steer, M. J. and David, J. P. R., "Extremely Low Excess Noise in InAs Electron Avalanche Photodiodes," IEEE Photonics Technology Letters 21(13), 866-868 (2009).

[14] Sun, W., Lu, Z., Zheng, X., Campbell, J. C., Maddox, S. J., Nair, H. P. and Bank, S. R., "High-gain InAs avalanche photodiodes," IEEE Journal of Quantum Electronics. 49(2), 154-161 (2013).

[15] Sandall, I. C., Zhang, S. and Tan, C. H., "Linear array of InAs APDs operating at $2 \mu \mathrm{m}, "$ Optics Express 21(22), 25780-25787 (2013).

[16] Marshall, A. R. J., Tan, C. H., Steer, M. J. and David, J. P. R., "Electron dominated impact ionisation and avalanche gain characteristics in InAs photodiodes," Appl. Phys. Lett. 93(11), 111107 (2008).

[17] Ker, P. J., Marshall, A. R. J., Krysa, A. B., David, J. P. R. and Tan, C. H., "Temperature dependence of leakage current in InAs avalanche photodiodes," IEEE Journal of quantum electronics 47(8), 1123-1128 (2008).

[18] "InAs photovoltaic detectors P10090 Series," Hamamatsu Photonics K. K., Hamamatsu, Japan (August 2014). http://www.hamamatsu.com/resources/pdf/ssd/p10090-01_etc kird1099e.pdf

[19] "J12 Indium arsenide detectors," Teledyne Judson Technologies LLC, USA. (March 2003). http://www.judsontechnologies.com/files/pdf/InAs_shortform_Mar2003.pdf

[20] Taylor, J., "Infrared Training Notes - level 1," Land Infrared Int. Ltd., UK, (14 January 2008). http://www.aumico.it/prodotti/files/117.pdf

[21] Saunders, P., "General interpolation equations for the calibration of radiation thermometers," Metrologia. 34(3), 201210 (1997).

[22] Saunders, P., "Uncertainty Arising from the Use of the Mean Effective Wavelength in Realizing ITS-90," Proc. AIP 684, 639-644 (2002). 